\title{
Cannabis in the emergency department: The impact of legalization on cannabis and opioid- related presentations
}

\author{
Matthew Yeung, Kevin Janz, Colin Weaver, Rebecca Saah-Haines, Eddy Lang
}

\begin{abstract}
On October 17th, 2018, non-medical cannabis became legal to adults for the first time in Canada. This has created a previously unseen opportunity to investigate the effects of recreational cannabis legalization in a developed country, particularly on strained Emergency Departments (EDs). Secondly, reports within the United States have suggested state-level legalization of cannabis, both recreationally and medically, has resulted in a decrease of opiaterelated presentations. Given the pressure of the opiate crisis on healthcare resources, we sought to examine if this trend was present in Alberta. Objectives: The current study aims to identify if presentation patterns in adult and pediatric populations have changed when comparing pre- and postlegalization periods, and if rural-urban disparities exist. We also aim to identify if the legalization of cannabis is correlated with a reduction in opiate-related ED presentations. Lastly, we aimed to address the aforementioned objectives in the context of telehealth by examining calls to poison control and HealthLink within Alberta. Methods: Retrospective data was collected from the National Ambulatory Care Reporting System, HealthLink, and Poison and Drug Information Service. Extraction is currently in progress, and we expect to include 20000 records and 12000 calls. An interrupted time-series analysis will be completed, allowing for a comparison of trends pre- and post-legalization. Participants have been identified based on International Disease Classifications for cannabis and opiate-related injury. Commonly reported injuries will be clustered to identify changes in injury patterns. Data was collected from October 1st 2013 up to May 31st, 2019 for all EDs within Alberta. Preliminary results suggest the legalization of cannabis initially led to a dramatic increase in ED presentations, followed by a return to pre-legalization volume. HealthLink data suggests a different trend, with steadily increasing calls in the months prior to legalization, followed by stabilization. Cannabis legalization is also correlated with a decrease in post-legalization opiaterelated calls $(r=-0.51, p=0.01)$. Overall, national legalization of cannabis appears to be responsible for a short period of increased ED usage, but does not appear to have longlasting effects on healthcare resource utilization. Differences are apparent between telehealth service and ED use.
\end{abstract}

Cite as: Yeung M, Janz K, Weaver C, Saah-Haines R, Lang E. 2019. Cannabis in the emergency department: The impact of legalization on cannabis and opioid-related presentations. Alberta Academic Review, Vol 2 (3) 6, CASCH Special Issue (not peer-reviewed), DOI: 10.29173/aar93. 